\title{
High-resolution transcription atlas of the mitotic cell cycle in budding yeast
}

\author{
Marina V Granovskaia ${ }^{1 \dagger}$, Lars J Jensen ${ }^{1,2+}$, Matthew E Ritchie ${ }^{3,4+}$, Joern Toedling ${ }^{5}$, Ye Ning ${ }^{6}$, Peer Bork', \\ Wolfgang Huber ${ }^{1,5}$, Lars M Steinmetz ${ }^{1 *}$
}

\begin{abstract}
Background: Extensive transcription of non-coding RNAs has been detected in eukaryotic genomes and is thought to constitute an additional layer in the regulation of gene expression. Despite this role, their transcription through the cell cycle has not been studied; genome-wide approaches have only focused on protein-coding genes. To explore the complex transcriptome architecture underlying the budding yeast cell cycle, we used 8 bp tiling arrays to generate a 5 minute-resolution, strand-specific expression atlas of the whole genome.

Results: We discovered 523 antisense transcripts, of which 80 cycle or are located opposite periodically expressed mRNAs, 135 unannotated intergenic non-coding RNAs, of which 11 cycle, and 109 cell-cycle-regulated proteincoding genes that had not previously been shown to cycle. We detected periodic expression coupling of sense and antisense transcript pairs, including antisense transcripts opposite of key cell-cycle regulators, like FAR1 and TAF2.

Conclusions: Our dataset presents the most comprehensive resource to date on gene expression during the budding yeast cell cycle. It reveals periodic expression of both protein-coding and non-coding RNA and profiles the expression of non-annotated RNAs throughout the cell cycle for the first time. This data enables hypothesisdriven mechanistic studies concerning the functions of non-coding RNAs.
\end{abstract}

\section{Background}

Genome-wide transcriptome analyses in humans [1-5], mouse [6], Drosophila melanogaster [7,8], Arabidopsis thaliana [9], and fission and budding yeast [10-12] have provided evidence for widespread expression of noncoding RNAs (ncRNAs) from intergenic as well as protein-coding regions (for example, antisense or intron-derived transcripts). ncRNAs have been implicated in regulation of chromatin structure, DNA methylation, transcription, translation, as well as RNA silencing and stability $[2,13-15]$.

Extensive transcription of intergenic regions and the antisense strands of hundreds of annotated protein-coding genes occurs in budding yeast, despite it lacking vestiges of the protein machinery required for microRNA or small interfering RNA processing [11,16-18]. It is not clear to what extent these RNAs are functional [19], but

\footnotetext{
* Correspondence: larsms@embl.de

+ Contributed equally

${ }^{1}$ EMBL - European Molecular Biology Laboratory, Department of Genome Biology, Meyerhofstr. 1, D-69117 Heidelberg, Germany
}

several have been shown to regulate transcription, acting through either transcriptional interference or epigenetic modifications. Examples of transcriptional interference are SRG1, a ncRNA transcribed in cis across the promoter of SER3 [20,21], and the antisense transcript of IME4 [22], whereas the antisense transcripts of PHO5 [23], PHO84 [24], transposable element Ty1 [25] and GAL10-ncRNA [26] function through epigenetic modification. For most newly discovered ncRNAs, the biological roles and mechanisms of action remain unknown. To unravel the functions of ncRNAs in yeast, it is informative to characterize them in the context of a robustly regulated and well-understood cellular process, such as the mitotic cell cycle, in which regulatory roles of ncRNAs have not been studied extensively.

The cell cycle orchestrates virtually all cellular processes - metabolism, protein synthesis, secretion, DNA replication, organelle biogenesis, cytoskeletal dynamics and chromosome segregation [27] - and diverse regulatory events depend on the maintenance of its periodicity. Between 400 and 800 periodically expressed
C Biomed Central

(c) 2010 Granovskaia et al.; licensee BioMed Central Ltd. This is an open access article distributed under the terms of the Creative Commons Attribution License (http://creativecommons.org/licenses/by/2.0), which permits unrestricted use, distribution, and reproduction in any medium, provided the original work is properly cited. 
protein-coding genes have been identified in the mitotic cell cycle and the genomic binding sites of transcription factors that control phase-specific expression of these genes have been mapped in genome-wide location analyses [28-30]. In addition to transcriptional regulation, strict timing of cell-cycle progression is ensured by post-translational regulation. This includes post-translational modifications, targeted protein degradation and indirect regulation via interactions with cell-cycle-regulated proteins [31].

To investigate the global cell cycle regulation of all transcripts, we measured high-resolution, strand-specific tiling microarray profiles of RNA expression during the Saccharomyces cerevisiae cell cycle. In contrast to previous studies [29,30], which only interrogated annotated features within the genome without resolving strand specificity, the fine spatial and temporal resolution of our dataset enabled us to look at the whole transcriptome on both strands, including non-coding RNAs (both away from coding genes and in antisense position), complex transcription architecture of protein-coding genes, alternative transcription start and polyadenylation sites, splicing, and differential regulation of sense and antisense transcripts. Our data reveal cellcycle-regulated non-coding genes, complex expression coupling between sense and antisense transcripts, as well as over 100 protein-coding genes that were not previously known to cycle.

\section{Results and discussion}

\section{Detecting periodic transcripts}

We monitored genome-wide cell-cycle-regulated expression at 5-minute intervals for up to three cell division cycles, using whole-genome tiling arrays [11]. The array is unique in interrogating every base pair in the genome on average six times and providing an 8 -bp resolution for strand-specific probes. Two independent synchronization methods were used in order to obtain synchronous cultures (see Materials and methods; Additional file 1). Late G1 phase arrest was induced by exposure of bar1 cells to alpha factor, and by raising the temperature to $38^{\circ} \mathrm{C}$ for temperature-sensitive $c d c 28-13$ mutant cells. Expression profiles for all genomic regions are provided in a database that is searchable by gene symbol or chromosomal coordinate [32].

To identify all transcribed sequences, we segmented along-chromosome expression profiles, applying an adaptation of the method described by Huber et al. [33] (see Materials and methods). In addition to protein-coding transcripts and infrastructural RNAs, we registered abundant expression of unannotated non-coding RNAs (Additional file 2). These unannotated expressed features comprise 523 antisense transcripts opposite protein-coding regions and 135 intergenic transcripts
(Additional file 3). The length distribution of ORFs in these unannotated transcripts is within the range that is expected by chance. Hence, we find no evidence for the unannotated transcripts to be protein coding.

The average segment levels from each time-point were analyzed for periodic expression by two different computational methods [34,35], as well as by visual inspection. The aim of this combination of methods was accurate and sensitive detection of cell-cycle-regulated transcripts (see Materials and methods). In order to validate our approach, we compared our gene list of periodic protein-coding genes to a benchmark set that comprised all known cell-cycle-regulated genes identified in single-gene experiments $[35,36]$. Our individual cdc28 and alpha-factor datasets were each better than most of the available ones [28-30] (Additional file 4). Furthermore, our combined list of periodic protein-coding genes, despite being based on just two experimental datasets, performed almost as well in identifying the benchmark set of genes as that of Gauthier et al. [37], which integrated all available genomic datasets of cellcycle-regulated genes performed to date (Additional file 4). Thus, our dataset and analysis method reproduced the previous data on cycling protein-coding genes.

Altogether, 598 periodic mRNAs, 37 cycling antisense RNAs, and 11 cycling intergenic transcripts were identified and ranked according to their peak time of expression (Figure 1; Additional file 5). Non-coding periodic transcripts were expressed in all cell-cycle phases (Figure 2; see Additional file 6 for the determination of the boundaries of the cell cycle phases). Overall, the peak times of antisense periodic expression were consistent with the waves of expression of periodic proteincoding genes [38]. To characterize the newly discovered periodic ncRNAs, we overlapped them with regions of conserved RNA secondary structure [39]. Despite their cell-cycle-regulated expression, the unannotated intergenic and antisense ncRNAs had little secondary structure (Additional file 6). Conversely, infrastructural ncRNAs, comprising tRNAs, rRNAs, small nuclear and small nucleolar RNAs, were highly structured but were not periodically expressed.

\section{Cell-cycle-regulated expression of unannotated non- coding RNAs}

Studies in mammalian cells have suggested that antisense RNAs could regulate gene expression of their sense counterparts, whereby sense and antisense transcripts often exhibit expression correlation patterns $[40,41]$ and overlap in opposite directionality [42]. We thus analyzed antisense RNAs in the context of the sense-antisense pairs (SAPs) of which they are a part. We categorized the pairs into four classes based on their expression coupling: 13 periodic antisense 


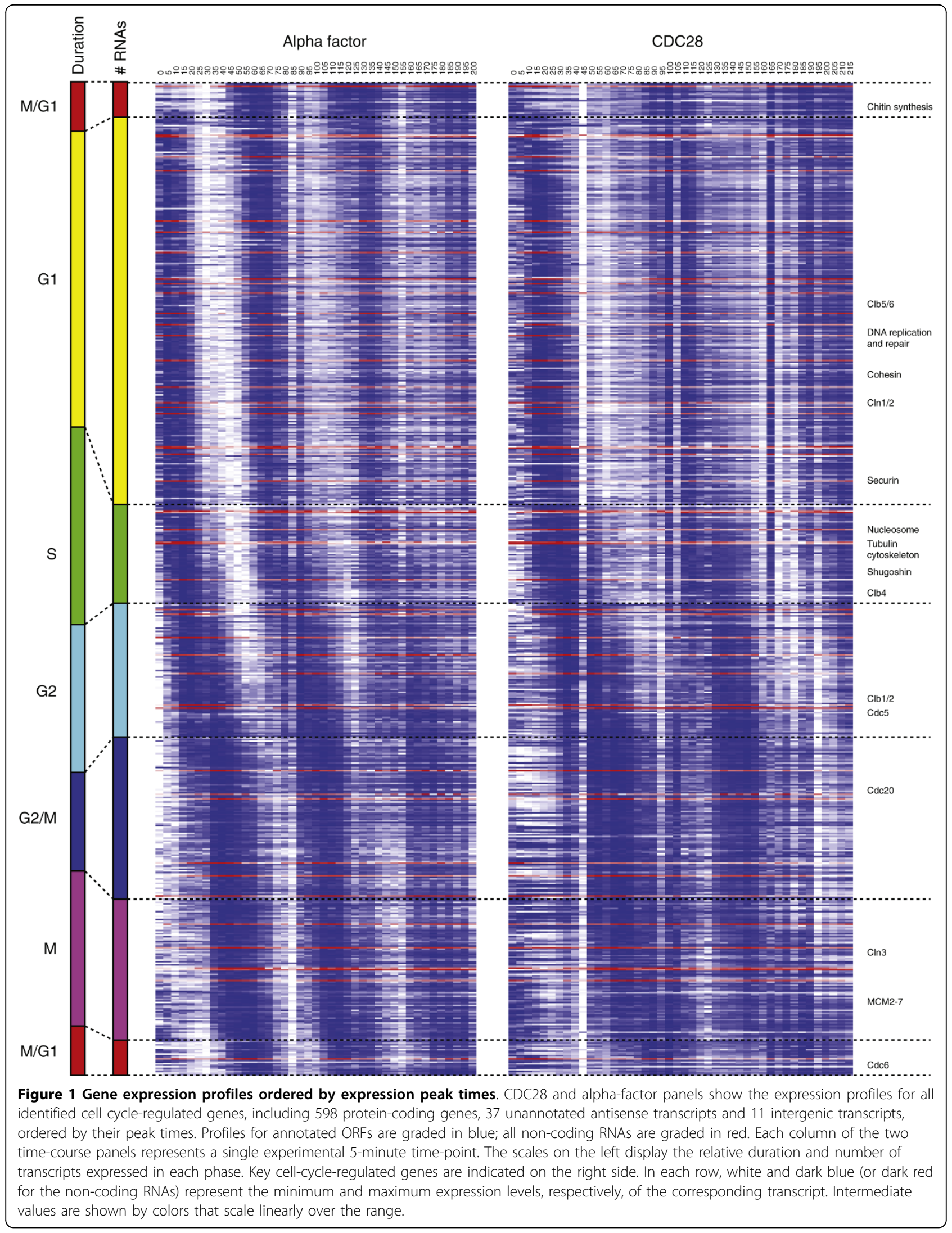




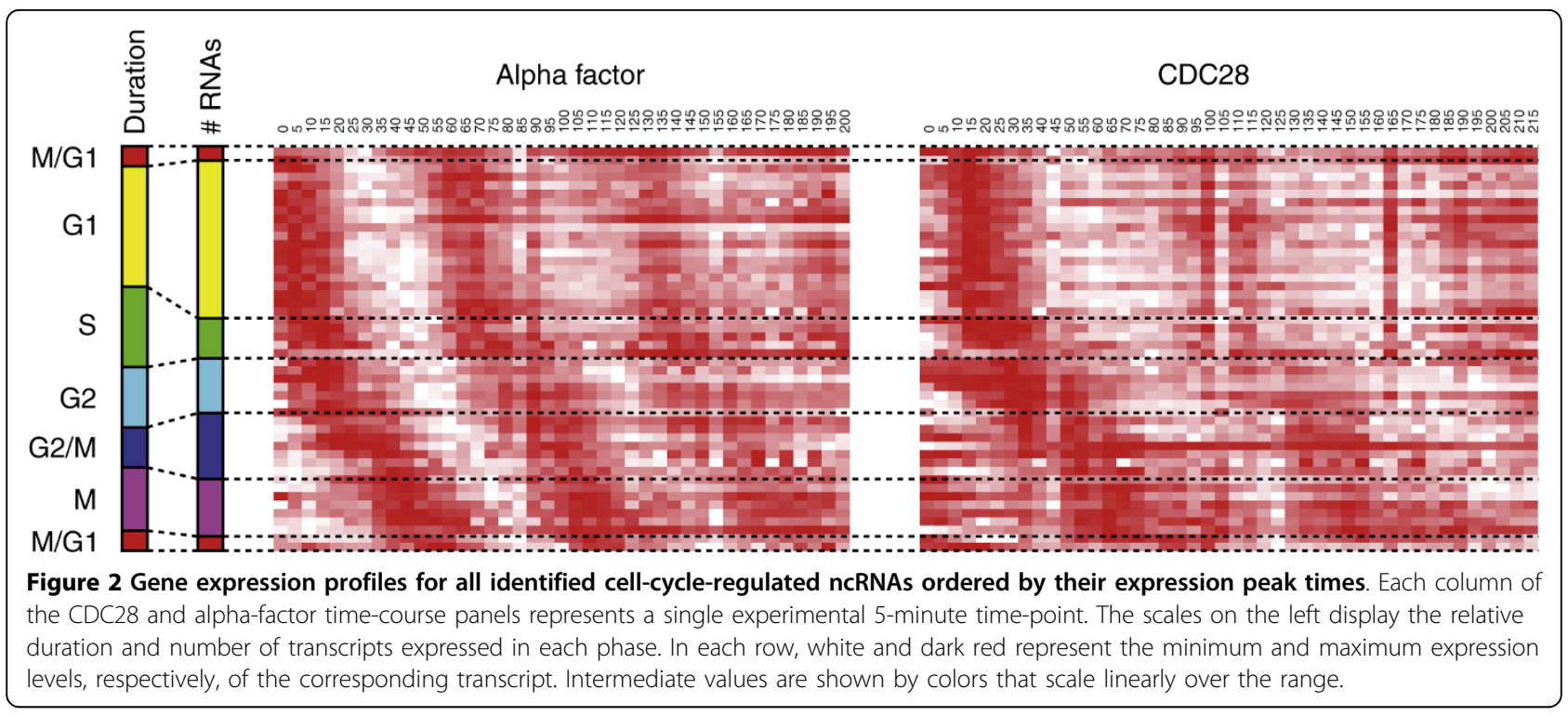

transcripts opposite periodic sense transcripts; 24 periodic antisense transcripts opposite non-periodic sense transcripts; 43 non-periodic antisense transcripts opposite periodic sense transcripts; and 443 non-periodic antisense and sense transcript pairs (Additional file 7).

The 13 periodic antisense transcripts opposite periodic sense transcripts were further subdivided based on the relative timing of expression of the sense and antisense transcripts. Considering the absolute difference between their expression peak times, two pairs (ALK1 and HSL1) cycle in-phase, whereas seven (CTF4, FAR1, HMS2, TAF2, TIP1, YNL300W and YPL162C) show anti-correlated expression (Additional file 8). Expression profiles of the other four SAPs (PRY3, YLR050C, YMR253C and $Y P L 230 W)$ had phase shifts between 0 and $\pi$.

Remarkably, several genes encoding important cell cycle regulators fall within the categories listed above (Figure 3a-c). Among them, FAR1 is important for mating pheromone-induced growth arrest and, together with cyclins CLN2 and CLN3, plays one of the key roles in the G1/S transition [43]. FAR1 is expressed at the M/ G1 transition and needs to be shut down in late G1 for the cell to pass the G1/S checkpoint. Its antisense RNA peaks starting from the late G1 phase and throughout the G1/S transition, when Far1 protein should not be present. TAF2, which is involved in transcription initiation, is expressed in late $\mathrm{M}$ and early G1 phase; its antisense transcript peaks in late $\mathrm{G} 1$ and further into $\mathrm{S}$ phase. The sense and antisense transcripts of CTF4, which shapes and maintains chromatin structure to ensure the passage through the S-phase checkpoint [44], are expressed in an anti-correlated manner, peaking in the G1/S and G2/M transitions, respectively. The CTF4 sense transcript appears to be transcribed from a bidirectional promoter shared with the antisense transcript of the neighboring gene, MSS18 (Additional files 6 and 9). Together these expression patterns suggest that some of the antisense transcripts may play a role in cell-cycle regulation.

We analyzed Gene Ontology (GO) categories of genes overlapped by antisense transcripts. Most of the proteincoding messages opposite the 37 periodic antisense transcripts $(13+24)$ fall into GO categories linked with the process of cell division, including cell wall and organelle organization and biosynthesis, regulation of transcription, signal transduction and protein modification, carbohydrate metabolic processes, and cell cycle (Additional file 10). Surprisingly, 15 of the 37 sense transcripts are of unknown function. We carried out a similar analysis for the 43 non-periodic antisense transcripts opposite periodic sense transcripts. As expected, most of these cycling sense messages fall into cell-cyclerelated GO categories, including genes involved in bud site selection and polarization (BUD9, GIC1), daughter cell separation from the mother (DSE2, CTS1), cell wall proteins, and so on (Additional file 7). Analysis of GO categories for the remaining 443 non-periodic SAPs did not show enrichment in any particular category, although almost a quarter of the genes have unknown function (Additional file 11).

We observed a statistically significant correlation $(P<$ 0.002; $5 \times 4$ contingency table; $\chi^{2}$ test) between the overlap patterns of the sense and antisense transcripts and the relationship of their expression profiles (Additional file 12). Altogether we distinguished five types of overlap within a given SAP: antisense transcript contains the transcribed message of its sense counterpart; the antisense transcript is contained within the sense 


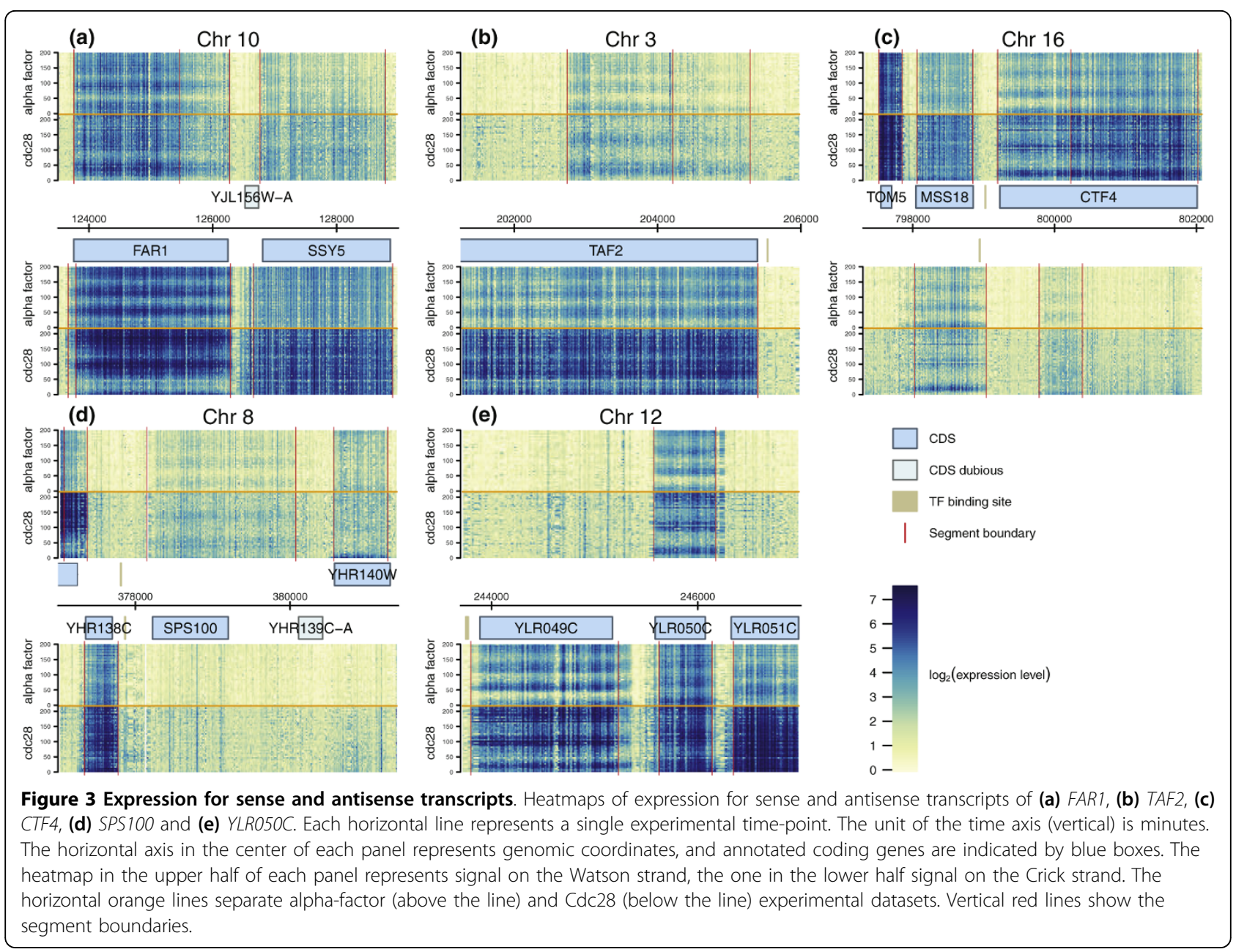

transcript; the antisense transcript overlaps either the 3' or the 5' end of its sense partner; and the antisense transcript overlaps two distinct sense transcripts. The following patterns of overlap were over-represented compared to what was expected by chance. In 8 out of 13 periodic antisense transcripts opposite periodic sense transcripts, the antisense transcript is mainly contained within the protein-coding message; 2 of these 8 cycle inphase, and 6 display opposite-phase expression. For 5 of 24. SAPs in which only the antisense transcript cycles, the antisense transcript contains the complete sense message, and for another 5, it overlaps 2 sense transcripts. In 15 of the 43 pairs in which only the sense message is cell cycle regulated, the antisense transcript overlaps the 5' end of the mRNA and in many cases extends further upstream.

To investigate sense and antisense expression in more detail, we also searched for putative TF binding sites (Additional file 6) and supported these predictions with the existing ChIP-chip data. TF binding site analyses are inherently non-strand-specific; however, our data on the temporal expression of the sense and antisense transcripts yield clues to the regulation of strand-specific expression. For example, ChIP-chip data and our motif analysis for FAR1 suggest binding of both the M-phase TF Mcm1 [45] and the G1/S TF SBF [46] within the region spanned by 600 bases before and after the transcript. This evidence for SBF regulation of FAR1 contradicts the timing of expression of the sense transcript since FAR1 is expressed at the M/G1 transition and needs to be shut down in late G1. Our data show lateG1-specific expression of the FAR1 antisense transcript, thus providing a putative explanation for the presence of the TF binding site for SBF. Overall, our analyses indicate that the cycling unannotated transcripts have binding sites for the same set of TFs that drive sense transcription during the cell cycle (Additional file 6).

Altogether, 135 unannotated intergenic transcripts were detected in our dataset. Of these, 11 oscillate with mitotic progression (Additional files 5; Additional file 13c). As for the antisense transcripts, their peak in expression follows the waves of excitation in mitotic 
progression observed for protein-coding genes [38]. To elucidate the role of these intergenic transcripts in cell cycle regulation, deletion strains for 10 of the 11 unannotated periodic transcripts were generated in both strain backgrounds. Growth curves of the deletion strains did not show significant lagging in cell doubling time after asynchronous growth in rich media for 28 hours at $30^{\circ} \mathrm{C}$ and $37^{\circ} \mathrm{C}$. Lack of phenotype is consistent with our previous observations for the unannotated intergenic transcripts detected from asynchronous culture [11]. This suggests that their deletion phenotypes have more subtle effects than those of many proteincoding genes.

\section{Cell cycle-regulated protein-coding genes}

Previous studies have identified a large number of annotated periodic transcripts. Compared to the integrated dataset of Gauthier et al. [37], our list contains 223 additional periodic protein-coding genes, of which 109 were also not identified by Pramila et al. [29] and Spellman et al. [30] (Figure 4; Additional file 14). Only 3 of the 109 have been shown to be periodically expressed in small scale experiments [47]. GOslim analysis [48] showed that the biological function is unknown for 35 of these 109 genes, whereas 41 perform functions directly or indirectly associated with the regulation of the cell cycle, such as organelle organization and biogenesis, cytoskeleton organization and biogenesis, ribosome biogenesis and assembly, and so on (Additional file 15).

Of the 598 periodically expressed protein-coding genes, just 7 contain an intron according to the Saccharomyces

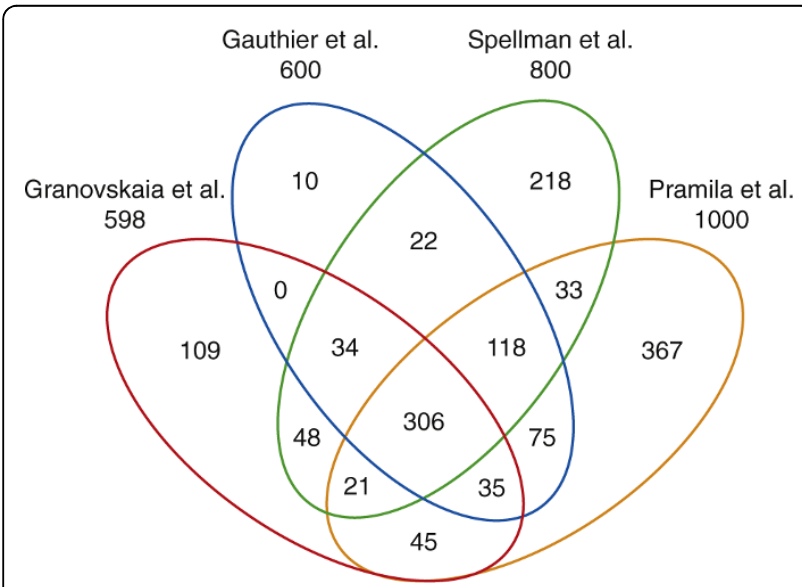

Figure 4 Venn diagram displays the overlap of our list of identified cell cycle-regulated protein-coding genes with the lists determined by the previous studies of Gauthier et al. [37], Pramila et al. [29], and Spellman et al. [30]. The overlap shows that we find an additional 223 genes not identified by Gauthier et al., among which 109 are unique to our dataset and were not previously defined by the other studies.
Genome Database annotation: CIN2, MOB1, PMI40, RFA2, SRC1, TUB1, and USV1. This is due to the fact that many of the budding yeast introns reside within genes that encode ribosomal proteins [48]. In addition, none of the introns in periodically expressed genes show signs of phase-specific splicing; hence, in contrast to meiosis in budding and fission yeast $[49,50]$, we see no evidence for a regulatory role of splicing in the mitotic cell cycle of budding yeast.

\section{Conclusions}

Our data provide 5-minute resolution strand-specific profiles of temporal expression during the mitotic cell cycle of S. cerevisiae, monitored for more than three complete cell divisions. The resulting atlas for the first time comprehensively maps the expression of non-annotated regions transcribed in mitotic circuitry, measures the expression coupling of protein-coding and non-coding transcript pairs and reveals strand specificity of transcription regulation. Furthermore, it unravels complex architectures of the mitotic transcriptome, such as splicing and alternative transcription start and polyadenylation sites, and extends the set of previously reported cell-cycle-regulated genes by 109 protein-coding genes.

The abundance of antisense expression across the genome raises the question of whether it represents opportunistic 'ripples of transcription' through active chromatin regions, or whether it is a regulated overlap between the transcripts [51]. An evolutionary analysis of genes with overlapping antisense partners across a number of eukaryotic genomes has indicated that the senseantisense arrangement is more highly conserved than expected if it were random 'leakage' of the transcription machinery [52].

Regulatory roles for a few antisense transcripts have been documented in yeast [20-25], yet it is still debated what proportion of ncRNAs are functional [19]. Our dataset reveals that most cycling antisense transcripts are located opposite genes with cell-cycle-related functions. Antisense transcripts may regulate the corresponding functional sense transcripts through several molecular mechanisms, which can be speculated from the mutual expression pattern of the two transcripts [53]. For example, transcriptional interference or antisense-dependent inhibitory chromatin remodeling may give rise to the anti-correlated expression of sense and antisense transcripts, as is observed for more than half of the 13 periodic SAPs. For the 24 cases where the antisense transcript cycles while the sense transcript is stably expressed, the periodic antisense transcript may putatively mask the sense transcript, thereby conferring periodic regulation at the level of translation. Through the same mechanism, the 43 stably expressed antisense transcripts may dampen stochastic fluctuation of sense 
messages by setting a threshold above which the sense expression must rise [53]. Alternatively, stably expressed antisense transcripts could mediate activatory chromatin remodeling that maintains the chromosomal region in a transcriptionally activatable/repressible state and thereby facilitate expression regulation of the periodic sense transcript. Indeed, more than one-third of the 43 stably expressed antisense opposite cell-cycle-regulated mRNAs overlap with the 5 ' UTRs. Altogether, the sense-antisense expression coupling may help to narrow down molecular mechanisms through which a specific antisense transcript exerts its function. Our high-resolution, unbiased expression atlas of the budding yeast cell cycle is thus a resource with which to unravel a potential additional level of the cell cycle regulatory circuit, as well as to study the periodic expression of protein-coding transcripts at a fine temporal and spatial resolution. The dataset provides a link between genomic approaches and hypothesis-driven mechanistic research with regard to the functions of ncRNAs.

\section{Materials and methods}

\section{Yeast strains and cell cycle synchronization}

W101 (50 ml; MATa ade2-1 trp1-1 leu2-3, 112 his3-11, 15 ura3 can1-100 [psi1]) background temperature-sensitive $c d c 28-13$ mutant $S$. cerevisiae strain K3445 (YNN553) was grown for approximately 8 to 10 hours in rich yeast-extract/peptone/dextrose (YPD) in a shaking water bath at $25^{\circ} \mathrm{C}$ and diluted in $3 \times 1.6$ liter cultures for overnight growth in an air incubator at $25^{\circ} \mathrm{C}$. The following morning the cultures of OD600 approximately 0.2 were mixed together, distributed into $45 \times$ $100 \mathrm{ml}$ samples and arrested in late G1 at START by shifting the temperature from $25^{\circ} \mathrm{C}$ to $38^{\circ} \mathrm{C}$. After 3.5 hours, the cells were transferred back to permissive temperature to re-initiate cell division and samples were collected every 5 minutes for 215 minutes (equal to more than two complete cell cycles). The cultures were centrifuged and snap-frozen in liquid nitrogen. The degree of synchrony was monitored by assessing the number of budding cells and measuring the bud size (Additional file 1). Nuclear position was determined by Hoechst staining with fluorescence microscopy (Additional file 16).

To arrest bar1 strain DBY8724 (MATa GAL2 ura3 bar1::URA3) [30] in G1 at START, alpha-factor pheromone was added to a final concentration of $600 \mathrm{ng} / \mathrm{ml}$. After 2 hours of arrest, cells were released by washing and recovered in fresh preconditioned medium to facilitate initiation of mitosis. Samples were collected every 5 minutes for 200 minutes (equal to three cell cycles). The degree of synchrony was monitored by assessing the number of budding cells. Nuclear position was determined by Hoechst staining with fluorescence microscopy.

\section{Total RNA extraction, poly(A)-RNA enrichment, cDNA synthesis and labeling}

Total RNA was isolated from the culture corresponding to each time-point by the standard hot phenol method [11]. Poly(A)-RNA was enriched from $1 \mathrm{mg}$ of total RNA by a single passage through the Oligotex Oligo-dT Column (Qiagen, Hilden, Germany). Poly(A)RNA was treated with RNase-free DNaseI (Ambion's Turbo DNA-free Kit, Foster City, CA, USA) for 25 minutes at $37^{\circ} \mathrm{C}$ according to the manufacturer's instructions and subsequently reverse transcribed to single-stranded cDNA for microarray hybridization. Each $200 \mu$ l reverse transcription reaction was carried out in duplicate and comprised $6 \mu \mathrm{g}$ of poly(A)-RNA, $3 \mu \mathrm{g}$ random hexamers (RH6), $1 \mu \mathrm{l}$ of $6 \mathrm{mg} / \mathrm{ml}$ Actinomycin D (ActD), $0.4 \mathrm{mM}$ dNTPs containing dUTP (dTTP:dUTP = 4:1), $40 \mu \mathrm{l} 5 \times$ first strand synthesis buffer (Invitrogen, Karlsruhe, Germany), $20 \mu \mathrm{l} 0.1 \mathrm{M}$ dithiothreitol (Invitrogen), and 1,600 units of SuperScript II (Invitrogen). The synthesis was carried out at $42^{\circ} \mathrm{C}$ for $1 \mathrm{~h}$ and 10 minutes, followed by reverse transcriptase inactivation at $70^{\circ} \mathrm{C}$ for 10 minutes. Poly(A)RNA and RNA in heteroduplex with cDNA were digested by a mixture of $3 \mu$ l of RNAseA/T cocktail (Ambion) and $3 \mu$ l of RNAseH (Invitrogen) for 15 minutes at $37^{\circ} \mathrm{C}$ followed by inactivation of the enzymes for 15 minutes at $70^{\circ} \mathrm{C}$. Replicate cDNA samples were further applied to the Affy Clean-up column (Affymetrix, Santa Clara, CA, USA), eluted together in $30 \mu \mathrm{DEPC}-\mathrm{H}_{2} \mathrm{O}$ and quantified. Purified cDNA (3.3 $\mu \mathrm{g}$ of each 5-minute time-point sample) was fragmented and labeled with WT Terminal Labeling Kit (Affymetrix) according to the manufacturer's instructions and then hybridized to tiling arrays.

\section{Genomic DNA preparation}

For DNA hybridization, both strains were grown in YPD media overnight to saturation in three biological replicates and whole-genomic DNA was purified using the Genomic DNA Kit (Qiagen). Genomic DNA (10 $\mu \mathrm{g})$ was digested to 25 to 100 base fragments with $0.2 \mathrm{U}$ of DNaseI (Invitrogen) in 1× One-Phor-All buffer (Pharmacia, Munich, Germany) containing $1.5 \mathrm{mM} \mathrm{CoCl}_{2}$ (Roche, Mannheim, Germany) for 3.5 minutes at $37^{\circ} \mathrm{C}$. After DNaseI inactivation by boiling for 10 minutes, the sample was 3' end-labeled in the same buffer by the addition of $1.5 \mu \mathrm{l}$ of Terminal Transferase (25 units/ $\mu$; Roche) and $1.5 \mu \mathrm{l} 10 \mathrm{mM}$ biotin-N6-ddATP (Molecular Probes, Karlsruhe, Germany) for 2 hours at $37^{\circ} \mathrm{C}$, and hybridized to the tiling array. 


\section{Array design}

The array was designed in collaboration with Affymetrix (PN 520055), as described in David et al. [11]. Probe sequences were aligned to the genome sequence of $S$. cerevisiae strain S288c (Saccharomyces Genome Database of 7 August 2005). Perfect match probes were further analyzed.

\section{Probe normalization and segmentation}

The log-base 2 perfect match (PM) probe intensities from each array were background corrected and calibrated using the DNA reference normalization method described in Huber et al. [33], which was applied separately to both datasets, cdc28 and alpha-factor.

To determine the transcript boundaries in the combined dataset, a piece-wise constant model was fitted to the normalized intensities of the unique probes ordered by genomic coordinates. The basic model described in Huber et. al. [33] was modified to allow time-pointdependent levels. The normalized intensities $\left(z_{j k}\right)$ were modeled as:

$$
z_{j k}=\mu_{s k}+\varepsilon_{j k} \text { for } t_{S} \leq j \leq t_{S+1}(1)
$$

where $\mu_{s k}$ is the array-specific level of the $s$-th segment, $\varepsilon_{j k}$ are the residuals, $j=1,2, ., n$ indexes the probes in ascending order along the chromosome, $k$ indexes the time-point (array), $t_{2}, ., t_{S}$ parameterize the segment boundaries $\left(t_{1}=1\right.$ and $\left.t_{S+1}=n+1\right)$ and $S$ is the total number of segments. Model 1 was applied separately to each strand of each chromosome. For each chromosome, $S$ was chosen such that the average segment length was 1,250 nucleotides. Change-points were estimated using a dynamic programming algorithm implemented in the tilingArray package [33].

After segmentation, the average of the probe signals within the segment boundaries was calculated for each time point. A table of segment levels is available from the supplementary materials webpage [32].

To estimate a threshold for expression, the average level over both datasets was calculated for each segment. Segments not overlapping annotated, transcribed features were used to estimate the background level as follows. A normal distribution was fit in order to determine a threshold at which the estimated false discovery rate was $0.1 \%$ [11]. For the mean of the normal distribution, we used the midpoint of the shorth (the shortest interval that covers half of the values), for the variance, the empirical variance of the lowest $99.9 \%$ of the data. Segments whose level fell below this threshold were considered not expressed.

Segments were then assigned to different categories depending on how they overlapped with annotated features as described in David et al. [11], with the difference of re-naming the unannotated isolated features to the unannotated intergenic. Expression values for each annotated feature were calculated as weighted averages of the overlapping segments on the same strand.

\section{Detection of periodic genes}

We used a combination of three approaches to identify periodically expressed segments and annotated features based on the cdc28 and alpha-factor datasets: the method of Ahdesmaki et al. [34], which calculates Pvalues for a robust nonparametric version of Fisher's gtest $[54,55]$, the permutation-based method of de Lichtenberg et al. [35], which scores genes based on both the magnitude of regulation and the periodicity of profile, and by systematic visual inspection. For the two computational methods, score cutoffs were determined based on comparison with existing benchmark sets of 113 known cycling genes identified in single-gene studies [47]. A combined list of cycling transcripts was compiled that contains all transcripts identified as cycling by at least two of the three methods. The peak time of expression for each transcript was calculated as percentage of the cell cycle duration as previously described [35]. To determine the length of the cell cycle in each experiment, the period length was optimized to fit the expression profiles for selected genes from the benchmark set.

\section{Analysis of protein-coding potential}

To test if the ncRNAs are likely to be novel protein-coding genes, we extracted all ORFs within unannotated antisense and intergenic transcripts and compared their length distributions to what would be expected by chance. The length of an ORF was defined as the distance between a stop codon and the most upstream ATG codon. Two separate background distributions were used for antisense and intergenic transcripts, to take into account that these two types of ncRNAs have different sequence properties ( $k$-mer frequencies), because the former are located opposite of protein-coding genes whereas the latter are located within intergenic regions. For antisense transcripts, a set of sequences with the same length distribution was sampled from the genomic regions opposite other protein-coding genes. Opposite genomic regions with matched length distribution and sequence properties were used as a background for the unannotated intergenic RNAs. The ORF length distributions observed for the antisense and intergenic transcripts were not statistically significantly different from their respective background distributions according to the Kolmogorov-Smirnov test. 


\section{Transcription factor binding sites analysis}

We used the TAMO suite [56] to identify the TFs that preferentially bind to regulatory regions of periodic noncoding transcripts. We systematically searched for binding motifs that were significantly overrepresented for the region, spanning from $-600 \mathrm{bp}$ upstream up to +600 bp downstream of 37 periodic unannotated antisense and 11 intergenic transcripts of interest, relative to a background set composed of all transcripts detected in the alpha-factor experiment. A benchmark set comprised 113 genes whose transcription was reported as cell cycle regulated in single-gene studies previously [47], whereas the lowest scoring 252 non-periodic antisense transcripts from the alpha-factor induced arrest dataset served as a negative control. We also performed de novo motif discovery on these sequences, using the combination of methods contained in the TAMO software suite. This analysis revealed no significantly overrepresented sequence motifs. We then searched for the putative TF binding sites that matched the position-specific score matrices from MacIsaac [57,58].

\section{Analysis of RNA secondary structure conservation}

We investigated the overlap between transcripts and genomic regions with conserved secondary structure [39]. We used Steigele et al.'s [39] regions for cutoff 0.5. The regions were remapped to the current genome assembly using Exonerate (requiring 100\% identity). The regions are strand-specific and overlap with these regions was also considered in a strand-specific way.

\section{Deletion strains of the periodic unannotated intergenic transcripts}

We generated deletion strains with the help of PCRbased technology as described on the Stanford Yeast Deletion webpage [59] using a set of up- and downstream primers flanking the defined periodic unannotated sequence listed in Additional file 5. The growth of deletion strains was monitored in liquid media using GENios automatic microplate readers (TECAN).

Additional file 1: A table providing control data on the synchronous division of the yeast cells. Excel sheet 1 contains a table of the number and percentage of budded cells and dividing nuclei over time with the progression of the cell cycle; sheet 2 contains a chart of these data.

Additional file 2: A figure showing categories of expressed segments. The pie chart shows the categories and the numbers of all identified transcribed segments. The unassigned categories encompass the segments that did not meet filter criteria and were excluded from further analyses [11]; correspondingly, the filtered categories are those that did pass the filtering criteria.

Additional file 3: A table listing antisense and novel intergenic transcripts identified in our study. Excel sheet 1 is a table of all 523 antisense transcripts, characterized by their genomic position, length and overlapping sense feature; sheet 2 is a table of all 135 unannotated intergenic transcripts, categorized by genomic position and length. Cycling intergenic transcripts are highlighted in sheet 2.
Additional file 4: A figure showing a comparison of our dataset with the published datasets on the cell cycle in yeast. Three ROC-like plots compare: (a) our combined dataset with that of Gauthier et al. [37]; (b) our cdc28 dataset with the other Cdc28 datasets of Spellman et al. [30] and Cho et al. [28]; (c) our alpha-factor dataset with the existing alphafactor datasets of Spellman et al. [30] and Pramila et al. [29]. The fraction of the B1 benchmark set genes identified by the various datasets is plotted as a function of gene rank. (a) Comparison of the method of de Lichtenberg et al. applied to our data (red line) with the comprehensive integrated dataset of Gauthier et al. (black line) [35]. The cross indicates our combined list, obtained by the combination of two computational methods of analyses, and curated manually. (b) Comparison of Cdc28 datasets. (c) Comparison of alpha factor-induced growth arrest datasets. The color code displays: light brown, Cho et al.; green, Spellman et al.; cyan and blue, Pramila et al.; black, Gauthier et al.; red, this study. The dotted line indicates random selection of genes.

Additional file 5: A table listing periodic protein-coding genes, antisense and unannotated intergenic transcripts. Excel sheet 1 lists 598 periodic ORFs identified in our dataset, sheet 2 lists 37 cycling antisense transcripts, and sheet 3 lists 11 periodic unannotated intergenic transcripts.

Additional file 6: A Word document providing supplemental data. The file provides additional information on the following sections: 1,

Determination of the boundaries of the cell cycle phases; 2, Conservation analysis of non-coding RNAs; 3, Analysis of upstream regulatory elements for periodic unannotated transcripts; 4, UTR lengths; 5, Divergently transcribed periodic transcripts.

Additional file 7: A table listing the categories of 37 periodic and 43 non-periodic antisense transcripts. Excel table sheet 1 lists 37 periodic antisense transcripts and sheet 2 lists 43 non-periodic antisense transcripts, each characterized by genomic position, length, overlapping sense feature, function of the opposite sense counterpart according to the Saccharomyces Genome Database, and peak time of expression (cycling 37 antisense transcripts only).

Additional file 8: A figure showing a comparison of the relative timing of expression within 13 periodic SAPs. We calculated the peak-time difference for the periodic sense and antisense transcripts within each of the 13 cycling SAPs for the alpha-factor and Cdc28 experiments separately. A difference of 0 corresponds to in-phase expression, whereas a difference of 50 corresponds to opposite-phase expression (180 degree phase shift). We observe a good correlation between the two experiments. The shape of the symbol shows how the sense-antisense counterparts overlap.

Additional file 9: A table listing pairs of pairs of divergent transcripts from a bidirectional promoter. Each transcript in a pair is characterized by the genomic location, category and gene name.

Additional file 10: A figure showing GO categories of the ORFs opposite cell-cycle-regulated antisense transcripts. The $x$-axis displays the number of genes and the $y$-axis shows the names of $G O$ categories.

Additional file 11: A figure showing $G O$ categories of 443 non-periodic ORFs opposite non-periodic antisense transcripts. The $x$-axis displays the number of genes and the $y$-axis shows the names of $G O$ categories.

Additional file 12: A contingency table for sense-antisense transcript overlap.

Additional file 13: A figure showing heatmaps of bi-directional expression of neighboring cell cycle-regulated genes that share transcription regulatory elements. (a) Two neighboring ORFs: TEL2 and ESP1. (b) ORF and an antisense transcript of the upstream protein-coding gene: SPT21 and antisense counterpart of YMR178W. (c) ORF and cycling unannotated intergenic transcript: MCD1and upstream cycling novel transcript. The heatmap plot is explained in the caption of Figure 3.

Additional file 14: A table listing the 109 periodic ORFs identified in our study.

Additional file 15: A figure showing GO categories of 109 periodic ORFs unique to our dataset. The $x$-axis displays the number of genes and the $y$-axis shows the names of $G O$ categories. 
Additional file 16: A figure showing Hoechst nuclear staining of dividing cdc28-ts mutant cells. Control data displaying synchronous division of the yeast cells along with the cell cycle progression. Each image represents a gallery of approximately 10 to 20 representative cells that were chosen, for the respective time-point, from different fields of view. Criteria of choice were sharpness of the image and visibility of the bud; besides these, we aimed for random selection.

\section{Abbreviations}

ChIP: chromatin immunoprecipitation; GO: Gene Ontology; ncRNA: noncoding RNA; ORF: open reading frame; SAP: sense-antisense pair; TF: transcription factor; UTR: untranslated region.

\section{Acknowledgements}

We thank Sandra Clauder-Muenster for technical assistance, Vladimir Benes and Tomi Baehr-Ivacevic from EMBL GeneCore Facility for technical advice, Yury Belyaev and Arne Seitz from EMBL-ALMF for help with image processing. This work was supported by grants to LMS from the National Institutes of Health and the Deutsche Forschungsgemeinschaft, to WH from the Human Frontier Science Program and to PB by the Bundesministerium fuer Bildung und Forschung (Nationales Genomforschungsnetz Foerderkennzeichen 01GS08169.)

\section{Author details}

${ }^{1} \mathrm{EMBL}$ - European Molecular Biology Laboratory, Department of Genome Biology, Meyerhofstr. 1, D-69117 Heidelberg, Germany. ${ }^{2}$ Novo Nordisk Foundation Center for Protein Research, Faculty of Health Sciences, University of Copenhagen, Blegdamsvej 3b, 2200 Copenhagen N, Denmark. ${ }^{3}$ Department of Oncology, University of Cambridge, CRUK Cambridge Research Institute, Li Ka Shing Centre, Robinson Way, Cambridge, CB2 ORE, UK. ${ }^{4}$ Bioinformatics Division, The Walter and Eliza Hall Institute of Medical Research, 1G Royal Parade, Parkville, Victoria 3052, Australia. ${ }^{5}$ EMBL European Bioinformatics Institute, Welcome Trust Genome Campus, Hinxton, Cambridge, CB10 1SD, UK. ' Plant Biochemistry Lab, Faculty of Life Sciences, University of Copenhagen, Thorvaldsensvej 40, 1871 Frederiksberg C, Denmark.

\section{Authors' contributions}

MVG and LMS designed research; MVG performed research; YN contributed to research; MVG, MER, LJJ, JT, WH and LMS analyzed data; MVG, LJ, MER, $\mathrm{WH}$ and LMS wrote the paper; WH, PB and LMS supervised research. The authors declare that they have no conflict of interest.

Received: 16 September 2009 Revised: 21 December 2009 Accepted: 1 March 2010 Published: 1 March 2010

\section{References}

1. Kampa D, Cheng J, Kapranov P, Yamanaka M, Brubaker S, Cawley S, Drenkow J, Piccolboni A, Bekiranov S, Helt G, Tammana H, Gingeras TR: Novel RNAs identified from an in-depth analysis of the transcriptome of human chromosomes 21 and 22. Genome Res 2004, 14:331-342.

2. Kapranov P, Cheng J, Dike S, Nix DA, Duttagupta R, Willingham AT, Stadler PF, Hertel J, Hackermuller J, Hofacker IL, Bell I, Cheung E, Drenkow J, Dumais E, Patel S, Helt G, Ganesh M, Ghosh S, Piccolboni A

Sementchenko V, Tammana H, Gingeras TR: RNA maps reveal new RNA classes and a possible function for pervasive transcription. Science 2007 316:1484-1488

3. Penn SG, Rank DR, Hanzel DK, Barker DL: Mining the human genome using microarrays of open reading frames. Nat Genet 2000, 26:315-318.

4. Schadt EE, Edwards SW, GuhaThakurta D, Holder D, Ying L, Svetnik V, Leonardson A, Hart KW, Russell A, Li G, Cavet G, Castle J, McDonagh P, Kan Z, Chen R, Kasarskis A, Margarint M, Caceres RM, Johnson JM, Armour CD, Garrett-Engele PW, Tsinoremas NF, Shoemaker DD: A comprehensive transcript index of the human genome generated using microarrays and computational approaches. Genome Biol 2004, 5:R73.
5. Yelin R, Dahary D, Sorek R, Levanon EY, Goldstein O, Shoshan A, Diber A, Biton S, Tamir Y, Khosravi R, Nemzer S, Pinner E, Walach S, Bernstein J, Savitsky K, Rotman G: Widespread occurrence of antisense transcription in the human genome. Nat Biotechnol 2003, 21:379-386.

6. Kiyosawa H, Yamanaka I, Osato N, Kondo S, Hayashizaki Y: Antisense transcripts with FANTOM2 clone set and their implications for gene regulation. Genome Res 2003, 13:1324-1334.

7. Hild M, Beckmann B, Haas SA, Koch B, Solovyev V, Busold C, Fellenberg K, Boutros M, Vingron M, Sauer F, Hoheisel JD, Paro R: An integrated gene annotation and transcriptional profiling approach towards the full gene content of the Drosophila genome. Genome Biol 2003, 5:R3.

8. Stolc V, Gauhar Z, Mason C, Halasz G, van Batenburg MF, Rifkin SA, Hua S, Herreman T, Tongprasit W, Barbano PE, Bussemaker HJ, White KP: A gene expression map for the euchromatic genome of Drosophila melanogaster. Science 2004, 306:655-660.

9. Yamada K, Lim J, Dale JM, Chen H, Shinn P, Palm CJ, Southwick AM, Wu HC, Kim C, Nguyen M, Pham P, Cheuk R, Karlin-Newmann G, Liu SX, Lam B, Sakano H, Wu T, Yu G, Miranda M, Quach HL, Tripp M, Chang CH, Lee JM, Toriumi M, Chan MM, Tang CC, Onodera CS, Deng JM, Akiyama K, Ansari $Y$, et al: Empirical analysis of transcriptional activity in the Arabidopsis genome. Science 2003, 302:842-846.

10. Wilhelm BT, Marguerat S, Watt S, Schubert F, Wood V, Goodhead I, Penkett CJ, Rogers J, Bahler J: Dynamic repertoire of a eukaryotic transcriptome surveyed at single-nucleotide resolution.. Nature 2008, 453:1239-1243.

11. David L, Huber W, Granovskaia M, Toedling J, Palm CJ, Bofkin L, Jones T, Davis RW, Steinmetz LM: A high-resolution map of transcription in the yeast genome. Proc Natl Acad Sci USA 2006, 103:5320-5325.

12. Dutrow N, Nix DA, Holt D, Milash B, Dalley B, Westbroek E, Parnell TJ, Cairns BR: Dynamic transcriptome of Schizosaccharomyces pombe shown by RNA-DNA hybrid mapping. Nat Genet 2008, 40:977-986.

13. Mattick JS, Makunin IV: Non-coding RNA. Hum Mol Genet 2006, 15(Spec No 1):R17-29.

14. Mattick JS, Gagen MJ: The evolution of controlled multitasked gene networks: the role of introns and other noncoding RNAs in the development of complex organisms. Mol Biol Evol 2001, 18:1611-1630.

15. Wassenegger M: RNA-directed DNA methylation. Plant Mol Biol 2000, 43:203-220

16. Miura F, Kawaguchi N, Sese J, Toyoda A, Hattori M, Morishita S, Ito T: A large-scale full-length cDNA analysis to explore the budding yeast transcriptome. Proc Natl Acad Sci USA 2006, 103:17846-17851.

17. Nagalakshmi U, Wang Z, Waern K, Shou C, Raha D, Gerstein M, Snyder M: The transcriptional landscape of the yeast genome defined by RNA sequencing. Science 2008, 320:1344-1349.

18. Samanta MP, Tongprasit W, Sethi H, Chin CS, Stolc V: Global identification of noncoding RNAs in Saccharomyces cerevisiae by modulating an essential RNA processing pathway. Proc Natl Acad Sci USA 2006, 103:4192-4197.

19. Struhl K: Transcriptional noise and the fidelity of initiation by RNA polymerase II. Nat Struct Mol Biol 2007, 14:103-105.

20. Martens JA, Laprade L, Winston F: Intergenic transcription is required to repress the Saccharomyces cerevisiae SER3 gene. Nature 2004, 429:571-574.

21. Martens JA, Wu PY, Winston F: Regulation of an intergenic transcript controls adjacent gene transcription in Saccharomyces cerevisiae. Genes Dev 2005, 19:2695-2704.

22. Hongay CF, Grisafi PL, Galitski T, Fink GR: Antisense transcription controls cell fate in Saccharomyces cerevisiae. Cell 2006, 127:735-745.

23. Uhler JP, Hertel C, Svejstrup JQ: A role for noncoding transcription in activation of the yeast PHO5 gene. Proc Natl Acad Sci USA 2007, 104:8011-8016.

24. Camblong J, Iglesias N, Fickentscher C, Dieppois G, Stutz F: Antisense RNA stabilization induces transcriptional gene silencing via histone deacetylation in S. cerevisiae. Cell 2007, 131:706-717.

25. Berretta J, Pinskaya M, Morillon A: A cryptic unstable transcript mediates transcriptional trans-silencing of the Ty1 retrotransposon in S. cerevisiae. Genes Dev 2008, 22:615-626. 
26. Houseley J, Rubbi L, Grunstein M, Tollervey D, Vogelauer M: A ncRNA modulates histone modification and mRNA induction in the yeast GAL gene cluster. Mol Cell 2008, 32:685-695.

27. Tyers M: Cell cycle goes global. Curr Opin Cell Biol 2004, 16:602-613

28. Cho RJ, Campbell MJ, Winzeler EA, Steinmetz L, Conway A, Wodicka L, Wolfsberg TG, Gabrielian AE, Landsman D, Lockhart DJ, Davis RW: A genome-wide transcriptional analysis of the mitotic cell cycle. Mol Cell 1998, 2:65-73.

29. Pramila T, Wu W, Miles S, Noble WS, Breeden LL: The Forkhead transcription factor $\mathrm{Hcm} 1$ regulates chromosome segregation genes and fills the S-phase gap in the transcriptional circuitry of the cell cycle. Genes Dev 2006, 20:2266-2278.

30. Spellman PT, Sherlock $G$, Zhang MQ, lyer VR, Anders $K$, Eisen MB, Brown PO, Botstein D, Futcher B: Comprehensive identification of cell cycleregulated genes of the yeast Saccharomyces cerevisiae by microarray hybridization. Mol Biol Cell 1998, 9:3273-3297.

31. de Lichtenberg U, Jensen TS, Brunak S, Bork P, Jensen LJ: Evolution of cell cycle control: same molecular machines, different regulation. Cell Cycle 2007, 6:1819-1825.

32. Tiling Array Data for Saccharomyces cerevisiae Cell Cycle Experiment. http://www.ebi.ac.uk/huber-srv/scercycle/.

33. Huber W, Toedling J, Steinmetz LM: Transcript mapping with high-density oligonucleotide tiling arrays. Bioinformatics 2006, 22:1963-1970.

34. Ahdesmaki M, Lahdesmaki H, Pearson R, Huttunen H, Yli-Harja O: Robust detection of periodic time series measured from biological systems. BMC Bioinformatics 2005, 6:117.

35. de Lichtenberg $U$, Jensen $L$, Fausboll A, Jensen TS, Bork P, Brunak S: Comparison of computational methods for the identification of cell cycle-regulated genes. Bioinformatics 2005, 21:1164-1171.

36. de Lichtenberg $U$, Jensen $L$, Brunak S, Bork P: Dynamic complex formation during the yeast cell cycle. Science 2005, 307:724-727.

37. Gauthier NP, Larsen ME, Wernersson R, de Lichtenberg U, Jensen LJ, Brunak S, Jensen TS: Cyclebase.org - a comprehensive multi-organism online database of cell-cycle experiments. Nucleic Acids Res 2008, 36 D854-859.

38. Lovrics A, Csikasz-Nagy A, Zsely IG, Zador J, Turanyi T, Novak B: Time scale and dimension analysis of a budding yeast cell cycle model. $B M C$ Bioinformatics 2006, 7:494.

39. Steigele S, Huber W, Stocsits C, Stadler PF, Nieselt K: Comparative analysis of structured RNAs in $S$. cerevisiae indicates a multitude of different functions. BMC Biol 2007, 5:25.

40. Havilio M, Levanon EY, Lerman G, Kupiec M, Eisenberg E: Evidence for abundant transcription of non-coding regions in the Saccharomyces cerevisiae genome. BMC Genomics 2005, 6:93.

41. Lehner B, Williams G, Campbell RD, Sanderson CM: Antisense transcripts in the human genome. Trends Genet 2002, 18:63-65.

42. Shendure J, Church GM: Computational discovery of sense-antisense transcription in the human and mouse genomes. Genome Biol 2002, 3: RESEARCH0044.

43. Vanoni M, Rossi RL, Querin L, Zinzalla V, Alberghina L: Glucose modulation of cell size in yeast. Biochem Soc Trans 2005, 33:294-296.

44. Warren CD, Eckley DM, Lee MS, Hanna JS, Hughes A, Peyser B, Jie C, Irizarry R, Spencer FA: S-phase checkpoint genes safeguard high-fidelity sister chromatid cohesion. Mol Biol Cell 2004, 15:1724-1735.

45. Simon I, Barnett J, Hannett N, Harbison CT, Rinaldi NJ, Volkert TL, Wyrick JJ, Zeitlinger J, Gifford DK, Jaakkola TS, Young RA: Serial regulation of transcriptional regulators in the yeast cell cycle. Cell 2001, 106:697-708.

46. Workman CT, Mak HC, McCuine S, Tagne JB, Agarwal M, Ozier O, Begley TJ, Samson LD, Ideker T: A systems approach to mapping DNA damage response pathways. Science 2006, 312:1054-1059.

47. Johansson $D$, Lindgren $P$, Berglund $A$ : A multivariate approach applied to microarray data for identification of genes with cell cycle-coupled transcription. Bioinformatics 2003, 19:467-473.

48. Hong EL, Balakrishnan R, Dong Q, Christie KR, Park J, Binkley G, Costanzo MC, Dwight SS, Engel SR, Fisk DG, Hirschman JE, Hitz BC, Krieger CJ, Livstone MS, Miyasato SR, Nash RS, Oughtred R, Skrzypek MS, Weng S, Wong ED, Zhu KK, Dolinski K, Botstein D, Cherry JM: Gene Ontology annotations at SGD: new data sources and annotation methods. Nucleic Acids Res 2008, 36:D577-581.
49. Juneau K, Palm C, Miranda M, Davis RW: High-density yeast-tiling array reveals previously undiscovered introns and extensive regulation of meiotic splicing. Proc Natl Acad Sci USA 2007, 104:1522-1527.

50. Bahler J: Cell-cycle control of gene expression in budding and fission yeast. Annu Rev Genet 2005, 39:69-94.

51. Ebisuya M, Yamamoto T, Nakajima M, Nishida E: Ripples from neighbouring transcription. Nat Cell Biol 2008, 10:1106-1113.

52. Dahary D, Elroy-Stein O, Sorek R: Naturally occurring antisense: transcriptional leakage or real overlap? Genome Res 2005, 15:364-368.

53. Lapidot M, Pilpel Y: Genome-wide natural antisense transcription: coupling its regulation to its different regulatory mechanisms. EMBO Rep 2006, 7:1216-1222.

54. Ueda HR, Chen W, Adachi A, Wakamatsu H, Hayashi S, Takasugi T, Nagano M, Nakahama K, Suzuki Y, Sugano S, lino M, Shigeyoshi $Y$, Hashimoto S: A transcription factor response element for gene expression during circadian night. Nature 2002, 418:534-539.

55. Wichert S, Fokianos K, Strimmer K: Identifying periodically expressed transcripts in microarray time series data. Bioinformatics 2004, 20:5-20.

56. Gordon DB, Nekludova L, McCallum S, Fraenkel E: TAMO: a flexible, objectoriented framework for analyzing transcriptional regulation using DNAsequence motifs. Bioinformatics 2005, 21:3164-3165.

57. Harbison CT, Gordon DB, Lee TI, Rinaldi NJ, Macisaac KD, Danford TW Hannett NM, Tagne JB, Reynolds DB, Yoo J, Jennings EG, Zeitlinger J, Pokholok DK, Kellis M, Rolfe PA, Takusagawa KT, Lander ES, Gifford DK, Fraenkel E, Young RA: Transcriptional regulatory code of a eukaryotic genome. Nature 2004, 431:99-104.

58. Maclsaac KD, Wang T, Gordon DB, Gifford DK, Stormo GD, Fraenkel E: An improved map of conserved regulatory sites for Saccharomyces cerevisiae. BMC Bioinformatics 2006, 7:113.

59. Yeast Deletion Webpage. [http://www-sequence.stanford.edu/group/ yeast_deletion_project/deletions3.html].

doi:10.1186/gb-2010-11-3-r24

Cite this article as: Granovskaia et al: High-resolution transcription atlas of the mitotic cell cycle in budding yeast. Genome Biology 2010 11:R24.

\section{Submit your next manuscript to BioMed Central and take full advantage of:}

- Convenient online submission

- Thorough peer review

- No space constraints or color figure charges

- Immediate publication on acceptance

- Inclusion in PubMed, CAS, Scopus and Google Scholar

- Research which is freely available for redistribution

Submit your manuscript at www.biomedcentral.com/submit
C Biomed Central 